\title{
Mean-Variance Versus Expected Utility in Dynamic Investment Analysis*
}

\author{
Yonggan Zhao William T. Ziemba \\ yonggan.zhao@commerce.ubc.ca ziemba@interchange.ubc.ca \\ (604) $822-2822$ \\ (604) $261-1343$
}

Faculty of Commerce and Business Administration

University of British Columbia

May 4, 2000

${ }^{*}$ This research was partially supported by NSERC. 


\title{
Mean-Variance Versus Expected Utility in Dynamic Investment Analysis
}

\begin{abstract}
This paper derives the mean-variance efficient frontier and optimal portfolio policies for a dynamic investment model. In the absence of arbitrage opportunities, the optimal expected portfolio value can be identified through the state price density in a frictionless market using martingale analysis. The efficient frontier for the dynamic model is linear in the space of the standard deviation and the expected value of the terminal portfolio in the presence of a riskless asset as in the static mean-variance case. A replication procedure is developed to obtain the optimal portfolio policies using a partial differential equation. A closed form solution is derived if asset prices jointly follow a multidimensional geometric Brownian motion. A comparison is made between the optimal policies of the expected utility approach and a mean-variance analysis in the continuous time setting. For investors interested in the mean-variance criterion, we discuss and derive the optimal choice of target wealth that maximizes the probability that the mean-variance analysis outperforms the expected utility approach.
\end{abstract}


MEAN-VARIANCE ANALYSiS DEVELOPED by Markowitz (1952) has presented a perfect balance between elegance and simplicity. Compared to the expected utility models, it offers a highly intuitive explanation for diversification and a relatively simple computational procedure. However, most discussions of mean-variance analysis are restricted to a static model which means that investors can only make a decision at the beginning and have to wait for the results at the end of horizon without adjusting the portfolio weights. This seems to be awkward for mean-variance analysis compared to versatile dynamic (multiperiod or continuous time) models that maximize expected utility. Tobin (1958) showed that the mean-variance model is consistent with von Neumann-Morgenstern postulates of rational behavior if the utility of wealth is quadratic. Since the quadratic utility function is increasing only up to some upper bound it introduces a stochastic control problem with a constraint on the wealth level which is difficult to solve using standard stochastic programming and stochastic control methodologies. In view of this weak point, this paper investigates and develops a dynamic version of mean-variance efficient frontier using martingale analysis. From the structure of the optimal portfolio value, relations between the mean-variance analysis and the expected utility approach are discussed.

Investment models can be categorized as those using the expected utility approach and those using the mean-variance analysis. The expected utility approach has been widely applied to both discrete and continuous time models. Merton $(1969,1971)$ led the way applying the stochastic control approach for dealing with utility maximization problem. With the definition of risk neutral probability, see Harrison and Kreps (1979), the martingale approach of dividing the problem into two simpler models has been extensively studied, see 
Harrison and Pliska (1981), Pliska (1986), Cox and Huang (1989), Zhao, Haussmann and Ziemba (2000). In these models, the static model identifies the possible optimal terminal wealth and the solution to the replication model, usually through partial differential equations, generates the optimal portfolio policy. With both methods, analytic solutions can be obtained for HARA utility functions. However, the martingale method seems to have more advantages than the stochastic control approach, because the former can deal properly with constraints on the wealth level.

The mean-variance analysis, as an important investment criterion, has not been properly incorporated in the continuous time models. The lack of this analysis in continuous time or multiperiod models has downgraded its practicality in developing dynamic investment models. This paper considers continuous time models with a mean-variance criterion. Applying martingale analysis, the efficient frontier and the optimal portfolio policies are derived assuming the absence of arbitrage and the existence of a riskless asset. If the price processes jointly have a Markovian structure, the optimal policies are obtained by solving a partial differential equation with boundary conditions. Furthermore, a closed form solution is derived if asset prices jointly follow a multidimensional geometric Brownian motion.

The comparisons made between the optimal values for the expected utility approach and the mean-variance analysis show that the latter achieves a better performance if the outcome of the market state price is around its mean value. On the other hand, the expected utility approach has better performance with outcomes beyond both tails of the state price which accommodates the risk aversion. Tobin (1958) pointed out that the investment proportions 
in the risky assets are independent of the utility function. Hakansson (1971) discussed and compared the optimal strategies obtained by the two criteria. By means of a three-asset portfolio strategy, Hakansson (1971) showed, not surprisingly, that the worst portfolio in the mean-variance sense performed better in the long run than most of the portfolios on the efficient frontier. Grauer (1981) performed a similar analysis by examining the similarity of the optimal portfolios derived by both strategies. Grauer and Hakasson (1993) compares the mean-variance and the quadratic utility approximation schemes for calculating the optimal portfolios in the discrete time dynamic investment model. Ziemba, Parkan and BrooksHill (1974) provided in the presence of a riskless asset a solution method that utilizes the theory of linear complementarity to find Tobin's linear efficient frontier independent of the investor's risk averse utility function, and stochastic programming to find an optimal portfolio once the utility function is specified. In this paper, we investigate from another angle the advantages of the mean-variance analysis over the expected utility approach. After obtaining the dynamic efficient frontier, an investor using the mean-variance criterion may ask the question: what is the optimal choice of target wealth that maximizes the probability that the mean-variance criterion outperforms the expected utility approach? To answer this question, we examine the relation of the optimal portfolio values for both mean-variance analysis and an expected utility strategy in terms of the state price. We provide a general method for calculating this probability and a closed form solution in the case of lognormal prices and logarithmic utility. A numerical example is presented which illustrates the practicality of the mean-variance analysis compared to the expected utility approach. 
In section 2, we discuss the asset price processes and the wealth evolution for given admissible strategies. Section 3 presents the efficient frontier and the comparison with the expected utility maximization. Section 4 deals with the solution of the optimal portfolio via a partial differential equation. In section 5, we derive a closed form solution assuming the market's primitive asset prices jointly follow a multidimensional geometric Brownian motion. Section 6 compares mean-variance analysis with the expected utility approach for dynamic investments. Section 7 concludes the paper.

\section{The Asset Price Model and The Wealth Process}

Assume a complete probability space $(\Omega, \mathcal{F}, P)$ and a time horizon $[0, T]$, where $T$ is a strictly positive real number. Let $z(t)=\left(z_{1}(t), \cdots, z_{n}(t)\right)^{\top}$ denote an $n$-dimensional standard Brownian motion, which generates a filtration $\mathbf{F}=\left\{\mathcal{F}_{t} \subseteq \mathcal{F} ; t \in[0, T]\right\}$, where $\mathcal{F}_{t}$ is the $\sigma$-field generated by $z(s), 0 \leq s \leq t$. A stochastic process $X(t)$ is called adapted to $\mathbf{F}$ if $X(t)$ is measurable with respect to $\mathcal{F}_{t}$. Consider a frictionless securities market with $n+1$ long-lived securities traded. Security $C$ is a locally riskless asset and securities $S_{i}$, $i=1, \cdots, n$, are risky assets. $\left(C, S_{1}, \cdots, S_{n}\right)$ are called the primitive assets for the market. The stochastic processes for the prices of these securities are

$$
\begin{aligned}
& d C(t)=r(t) C(t) d t, \quad C(0)=1 \\
& d S_{i}(t)=b_{i}(t) d t+\sum_{j=1}^{n} \sigma_{i j}(t) d z_{j}(t), \quad \forall i=1,2, \cdots, n .
\end{aligned}
$$

To make the problem a general Markovian diffusion process, $r(t), b_{i}(t)$ and $\sigma_{i j}(t)$ are allowed to be functions (nonrandom) of $C(t)$ and $\left\{S_{i}(t)\right\}$. For simplicity, we supress $C(t)$ and $\left\{S_{i}(t)\right\}$ 
whenever the expression is clear. Let $b(t)=\left(b_{1}(t), \cdots, b_{n}(t)\right)^{\top}$ and $\sigma(t)=\left(\sigma_{i j}(t)\right)_{n \times n}$ be an $n \times n$ matrix process. Assume that $b$ and $\sigma$ are Borel measurable and satisfiy the It $\hat{o}$ conditions and $\sigma(t)$ is a non-singular matrix.

A trading strategy is an $n+1$ - vector process, denoted by

$$
\left\{\alpha(t), \theta(t) \equiv\left(\theta_{1}(t), \cdots, \theta_{n}(t)\right)^{\top} ; t \in[0, T]\right\}
$$

where $\alpha(t)$ and $\theta_{i}(t)$ are the number of shares of the riskless asset and of the ith risky asset, respectively, and they are $\mathcal{F}_{t}$ - predictable processes. A self-financing strategy can be characterized as

$$
\alpha(t) C(t)+\theta(t)^{\top} S(t)=\alpha(0) C(0)+\theta(0) S(0)+\int_{0}^{t} \alpha(s) d C(s)+\int_{0}^{t} \theta(s)^{\top} d S(s)
$$

which ensures that the instantaneous portfolio return is a weighted sum of the asset returns. The interpretation is that the current portfolio value, given by the lefthand side of (2) equals the initial investment plus capital gains to date. We allow $(\alpha(t), \theta(t))$ to take negative values corresponding to shortsales, as well as positive values. A negative value for $\alpha(t)$ represents borrowing at the riskless rate $r(t)$. Negative values of $\theta(t)$ represent short sales of stocks by the investor, i.e., the stock is sold at time $t$, but not delivered until time $T$. If $W(t)$ is the portfolio value at time $t$, then

$$
\begin{aligned}
d W(t) & =\alpha(t) \cdot d C(t)+\theta(t)^{\top} \cdot d S(t) \\
& =r(t) W(t) d t+\theta(t)^{\top}[(b(t)-r(t) S(t)) d t+\sigma(t) d z(t)] .
\end{aligned}
$$

To make this stochastic differential equation well defined, we assume that an admissible 
trading strategy must satisfy the following conditions:

(i). $\int_{0}^{T}\left|\theta^{\top}(t)(b(t)-r(t) S(t))\right| d t<\infty, \quad$ P - a.s.

(ii). $\int_{0}^{T}\left\|\theta(t)^{\top} \sigma(t)\right\|^{2} d t<\infty, \quad$ P - a.s.

Assume that $\int_{0}^{T}|r(t)| d t<\infty, \int_{0}^{T}\left|b_{i}\right| d t<\infty, \int_{0}^{T}\left|\sigma_{i j}\right|^{2} d t<\infty, \quad P-a . s .$, and that the Novikov condition holds: $E \exp \left(\frac{1}{2} \int_{0}^{T}\left\|\sigma(t)^{-1}(b(t)-r(t) S(t))\right\|^{2} d t\right)<\infty$. A typical example is $b_{i}(t)=\bar{b}_{i}(t) S(t), \sigma_{i j}=S_{i} \bar{\sigma}_{i j}$, where $\bar{b}_{i}, \bar{\sigma}_{i j}$ are bounded functions of $t$. This implies that $\kappa=\sigma(t)^{-1}(b(t)-r(t) S(t))=\bar{\sigma}(t)^{-1}(\bar{b}(t)-r(t) \mathbf{1})$ is bounded; therefore, the Novikov condition holds.

Harrison and Kreps (1979) have shown that the existence of an arbitrage-free market is equivalent to the existence of a probability measure under which all prices of securities discounted at the riskless rate are martingales. An equivalent martingale measure $Q$ is a probability measure on $(\Omega, \mathcal{F})$ equivalent to $P$ such that all prices in units of the riskless asset are martingales with respect to the $\sigma$ - fields $\mathcal{F}_{t}$, i.e.

$$
E^{Q}\left(\frac{S_{i}(s)}{C(s)} \mid \mathcal{F}_{t}\right)=\frac{S_{i}(t)}{C(t)}, \quad \forall i=1,2, \cdots, n
$$

where $E^{Q}(\cdot)$ is the expectation operator taken under the probability measure $Q$.

With the setting of model (1), there exists a unique equivalent martingale measure which has the explicit expression

$$
\frac{d Q}{d P}=\exp \left\{-\int_{0}^{T} \kappa(s)^{\top} d z(s)-\frac{1}{2} \int_{0}^{T}\|\kappa(s)\|^{2} d s\right\}
$$

where $\kappa(t) \equiv \sigma(t)^{-1}(b(t)-r(t) S(t))$, called the market price of risk, and $\kappa$ satisfies the Novikov condition, $E\left(\exp \left\{\frac{1}{2} \int_{0}^{T}\|\kappa(s)\|^{2} d s\right\}\right)<\infty$, hence $E^{Q}\left(\mathbf{1}_{\Omega}\right)=1$, which implies 
there is no arbitrage and $Q$ is a probability measure. The exponential $P$ - martingale generated by $\kappa(t)$ is

$$
\eta(t) \equiv \exp \left\{-\int_{0}^{t} \kappa(s)^{\top} d z(s)-\frac{1}{2} \int_{0}^{t}\|\kappa(s)\|^{2} d s\right\}
$$

Utilizing Levy's characterization for Brownian motion yields that

$$
z^{Q}(t) \equiv z(t)+\int_{0}^{t} \kappa(s) d s
$$

is a standard Brownian motion under the probabilty measure $Q$. This result is usually referred to as Girsanov's theorem.

Under the Brownian motion $z^{Q}(t)$, the asset prices and the portfolio value can be rewritten as

$$
\begin{aligned}
& d S_{i}(t)=r(t) S_{i}(t) d t+\sum_{j=1}^{n} \sigma_{i j}(t) d z_{j}^{Q}(t) \\
& d W(t)=r(t) W(t) d t+\theta(t)^{\top} \sigma(t) d z^{Q}(t)
\end{aligned}
$$

which implies that all self-financed portfolio processes (including the primitive assets) have the same drift rate $r(t)$ under the $Q$ - Brownian motion. Conversely, it is also true that an $\mathcal{F}_{t}$ adapted martingale process is a portfolio process (meaning replicable) by the martingale representation theorem. 


\section{The Efficient Frontier}

\section{A. The Mean-Variance Model and Its Variant Versions}

The static mean-variance model may be formulated in two ways: minimizing variance subject to an expected wealth target or maximizing the expected wealth subject to a given level of risk, the variance. Both ways can trace out the efficient frontier by varying the corresponding parameters. Let $E(\cdot), V(\cdot)$, and $V^{2}(\cdot)$ stand for the operators of expected value, standard deviation, and the variance of a random variable in question. The dynamic mean-variance model is

$$
\begin{aligned}
\min _{\theta(\cdot), W(\cdot)} & V^{2}(W(T)) \\
\text { s.t. } & E(W(T)) \geq \mu, \\
& d W(t)=r(t) W(t) d t+\theta(t)^{\top} \sigma(t) d z^{Q}(t), \quad 0 \leq t \leq T,
\end{aligned}
$$

where $\mu$ is the expected wealth target. The stochastic control problem above with an irregular objective function and a constraint on the target level of expected wealth present a significant obstacle for obtaining solutions through stochastic control methodology. However, the martingale approach can greatly reduce the complexity of the problem. The problem can be divided to two submodels: a static model for identifying the optimal attainable wealth and a replication model for obtaining the optimal portfolio policies. We 
consider first the static problem

$$
\begin{array}{ll}
\min _{W} & V^{2}(W) \\
\text { s.t. } & E(W) \geq \mu \\
& E^{Q}\left(C^{-1}(T) W\right)=W_{0},
\end{array}
$$

where $W_{0}$ is the initial wealth and $E^{Q}(\cdot)$ is the expectation operator under the risk neutral probability. The optimal portfolio values for models (7) and (8) coincide to each other using a replication argument.

Since the operator $V^{2}(\cdot)$ is in the objective function of Model (8), a Kuhn Tucker solution procedure does not directly apply. However, a simpler version of Model (8) is given in Corollary 1.

Corollary 1: Assuming the existence of a riskless asset, Model (8) is equivalent to the model

$$
\begin{array}{rl}
\min _{W} & E\left(W^{2}\right)-\mu^{2} \\
\text { s.t. } & E(W)=\mu \\
& E^{Q}\left(C^{-1}(T) W\right)=W_{0} .
\end{array}
$$

To verify Corollary 1 , one needs only to show that, if $W^{*}$ is optimal for (8), then $E\left(W^{*}\right)=\mu$. In fact, if $E\left(W^{*}\right)>\mu$, then one can construct a portfolio $W^{* *}$ by setting a terminal portfolio to be $\frac{\mu}{E\left(W^{*}\right)} W^{*}$ plus the amount in the risk free asset. Obviously, $E\left(W^{* *}\right) \geq \mu$ and the martingale constraint is satisfied as well. Furthermore, $W^{* *}$ has a smaller variance than $W^{*}$, which contradicts the optimality of $W^{*}$. 


\section{B. The Optimal terminal Wealth}

Denote $\xi=C(T)^{-1} \eta(T)$ as the contingent state price. The Lagrangian of Model (9) is

$$
\mathcal{L}(W, \lambda, \rho)=E\left(W^{2}\right)-\mu^{2}-\lambda(E(W)-\mu)-\rho\left(E(\xi W)-W_{0}\right)
$$

where $\lambda$ and $\rho$ are the multipliers on the target expected wealth constraint and the martingale constraint, respectively. The extended Kuhn Tucker criteria are necessary and sufficient conditions due to the convexity of the objective function and the convexity of the feasible region. Theorem 1 characterizes the optimal value.

Theorem 1: The optimal portfolio value is a linear function of the state price $\xi$ with a negative slope if the target return is greater than the riskless rate. Furthermore, the optimal value is

$$
W=\frac{1}{2} \lambda+\frac{1}{2} \rho \xi
$$

where the multipliers are given by

$$
\begin{aligned}
\lambda & =\frac{2 \mu E\left(\xi^{2}\right)-2 W_{0} E(\xi)}{V^{2}(\xi)} \\
\rho & =\frac{2 W_{0}-2 \mu E(\xi)}{V^{2}(\xi)} .
\end{aligned}
$$

Proof of Theorem 1. Applying the Kuhn Tucker criteria yields

$$
\begin{array}{r}
2 W-\lambda-\rho \xi=0 \\
E(W)-\mu=0 \\
E(\xi W)-W_{0}=0
\end{array}
$$


which implies (10). Applying the operators $E(\cdot)$ and $E^{Q}(\cdot)$ to both sides of (10) yields

$$
\begin{gathered}
\lambda+\rho E(\xi)=\mu \\
\lambda E(\xi)+\rho E\left(\xi^{2}\right)=W_{0} .
\end{gathered}
$$

Solving (12) yields (11), which completes the proof of Theorem 1.

It is observed that the optimal portfolio is a linear function of the contingent state price, $\xi$, with non-positive slop. Hence, one can identify the optimal portfolio by obtaining the probability distribution of the contingent state price.

\section{The Efficient Frontier}

Let $\Psi$ be the standard deviation of the optimal portfolio of Model (9). Substituting the optimal multipliers $\lambda$ and $\rho$ in the optimal wealth expression in (10) proves the following Theorem 2.

Theorem 2: The relation of the optimal value and the wealth target is given by

$$
\Psi=\left\{\begin{array}{lc}
\frac{E(\xi)}{V(\xi)}\left(\mu-W_{0}(E(\xi))^{-1}\right) & \text { if } \mu \geq W_{0}(E(\xi))^{-1} \\
-\frac{E(\xi)}{V(\xi)}\left(\mu-W_{0}(E(\xi))^{-1}\right) & \text { if } \mu<W_{0}(E(\xi))^{-1} .
\end{array}\right.
$$

Proof of Theorem 2. By Theorem 1,

$$
E\left(W^{2}\right)=\frac{1}{2} E(W)+\frac{1}{2} E(\xi W) .
$$

Substituting $\lambda$ and $\rho$ into (10) yields

$$
E\left(W^{2}\right)-\mu^{2}=\frac{(E(\xi))^{2}}{V^{2}(\xi)}\left(\mu-E(\xi)^{-1}\right)^{2}
$$


which completes the proof of Theorem 2 .

Figure 1 depicts the feasible region of portfolio policies as shaded between the two lines in the mean-standard deviation space. The upward linear segment is the efficient

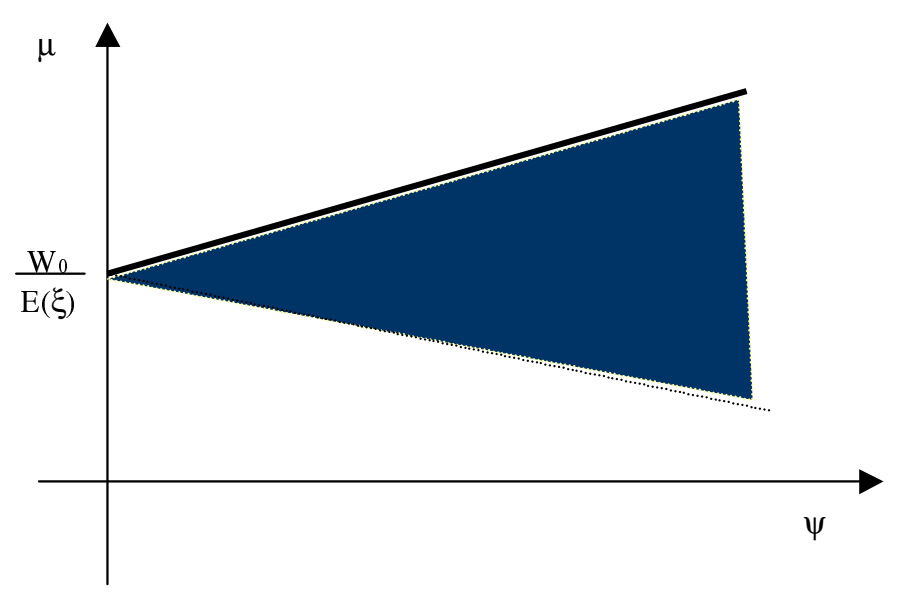

Figure 1: The Dynamic Mean-Variance Efficient Frontier

frontier and the downward linear segment the inefficient frontier; see Tobin (1958) and Ziemba et al (1974). The dynamic mean-variance model has a similar shape but with a different slope than the static mean-variance efficient frontier. The dynamic mean-variance efficient frontier is above that of the static mean-variance strategy since the set of selffinancing strategy contains the set of static strategies as a subset. Furthermore, the mean and the standard deviation of the contingent state price uniquely determine the meanvariance efficient frontier. 


\section{A Comparison with the Expected Utility Approach}

\section{A. The relation between the Terminal Portfolios}

Much research in the literature has been focused on determining which of the expected utility and the mean-variance analysis is favorable in making sound investment decisions. The optimal portfolio generated from a utility maximization is not on the mean-variance efficient frontier except in a few special instances: unless either a "carefully" chosen quadratic utility or joint normally distributed asset returns; see Samuelson (1970), and Ziemba and Vickson (1975) for other exceptions. However, investors and academic researchers do not accept these assumptions for practical use. For users of mean-variance analysis, the following question may be asked: what is the best choice of the target wealth such that the terminal portfolio has the maximum probability of being superior to the portfolio obtained from a utility maximization approach?

Let $W_{m}$ and $W_{u}$ be the terminal portfolio value for mean-variance analysis and an expected utility approach, respectively. $W_{m}$ is given by (10) and (11). It can be easily derived using a martingale argument that optimal value for the growth optimal strategy is

$$
W_{u}=U_{x}^{-1}\left(\lambda_{u} \xi\right)
$$

where $\lambda_{u}$ is the Lagrangian multiplier on the wealth constraint for given utility function $U(x)$ and $U_{x}^{-1}(\cdot)$ stands for the inverse function of the marginal utility of wealth; see Cox and Huang (1989). Figure 2 depicts the relation of the two optimal portfolio values in terms of the state price $\xi$. Since $U(x)$ is strictly increasing and concave, $W_{u}$ is a convex function 


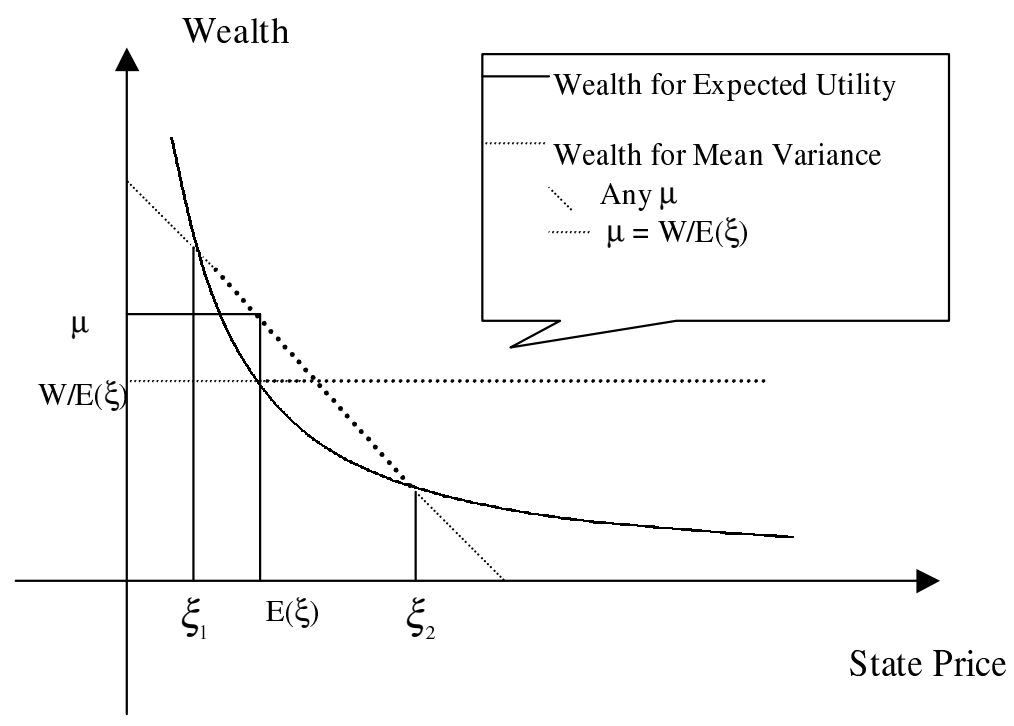

Figure 2: The Optimal Terminal Portfolio Values

of $\xi$. On the other hand $W_{m}$ is a straight line with negative slope. Therefore, there are two intersection points $\xi_{1}$ and $\xi_{2}$. Mean-variance analysis will be superior if the outcome of the state price $\xi$ occurs around the mean value $E(\xi)$ of the state price, as represented by the bold dotted line segments, and be inferior if the outcome is beyond one of the tails, $\xi_{1}$ or $\xi_{2}$

\section{B. Opportunities Superior to the Expected Utility Approach}

By varying $\mu$, investors can find the maximum probability that the mean-variance optimal portfolio will outperform the expected utility maximization portfolio. The maximum probability is given by solving the maximization problem

$$
\max _{\mu} \operatorname{Pr}\left\{\xi_{1} \leq \xi \leq \xi_{2} \mid \mu \geq W_{0} / E(\xi)\right\}
$$


where $\xi_{1}$ and $\xi_{2}, \xi_{1}<\xi_{2}$, are the two intersection points ${ }^{1}$ of $W_{u}$ and $W_{m}$ which satisfy the following transcendental equation,

$$
-2 U_{x}^{-1}\left(\lambda_{u} \xi\right)+\lambda+\rho \xi=0
$$

Since $\lambda$ and $\rho$ are functions of $\mu$, so are $\xi_{1}$ and $\xi_{2}$. Both the mean-variance analysis and expected utility approaches are considered as standard approaches for constructing optimal investment strategies. For mean-variance optimizers, an interesting question is how to set the target wealth level such that the mean-variance criterion will be superior to the expected utility approach with maximum probability. With appropriate conditions, we can calculate the optimal value $\mu$ and, therefore, the maximum probability. Let $\phi(x)$ be the density function of $\xi$. Assuming that there is a solution to (15) and that both $\xi_{1}(\mu)$ and $\xi_{2}(\mu)$ are differentiable with respect to $\mu$, then problem (15) becomes

$$
\max _{\mu} \int_{\xi_{1}}^{\xi_{2}} \phi(x) d x
$$

By the first order conditions, the optimal $\mu$ is given by

$$
\phi\left(\xi_{2}(\mu)\right) \xi_{2}^{\prime}(\mu)-\phi\left(\xi_{1}(\mu)\right) \xi_{1}^{\prime}(\mu)=0
$$

where "I" stands for the derivative.

\section{A Numerical Example}

Consider an investor having one dollar to invest between a riskless asset and a risky asset. The riskless interest rate for the period of March 7, 1997 to February 7, 2000 was

\footnotetext{
${ }^{1}$ Since $W_{u}$ is a convex function of the contingent state price $\xi$ and $W_{m}$ is linear in $\xi, W_{u}$ and $W_{m}$ intersect at exactly two points for a given $\mu<\infty$.
} 
about $r=0.05$ per annum, i.e., the riskless asset price $C(t)=e^{r t}$. The Dow Jones Industrial Average is the risky asset. After scaling the initial index level to a dollar, Figure 3 depicts the price dynamics of the Dow Jones Industrial Average for the period of March 7, 1997 to February 7, 2000.

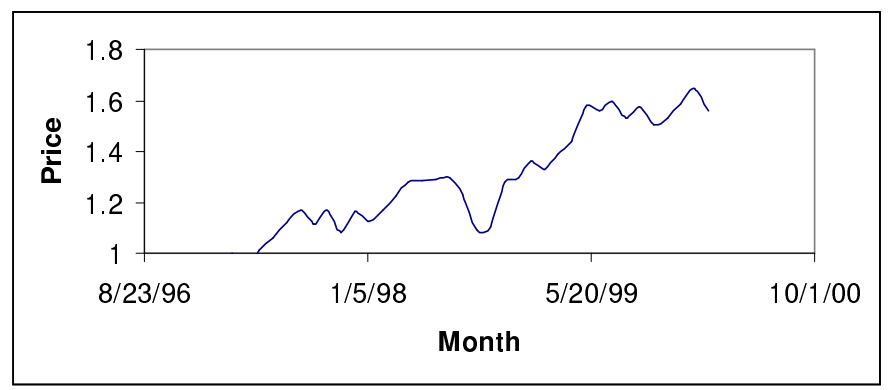

Figure 3: Scaled index level of DJIA.

Assuming that the price $S(t)$ of the DIJA follows the geometric Brownian motion

$$
d S(t)=b S(t) d t+\sigma S(t) d z(t)
$$

with estimated $b=0.1534$ and $\sigma=0.1863$. Let the investment horizon be one year, so $T=1$. The market price of risk $\kappa=(b-r) / \sigma=0.5551$ and, by definition, the state price $\xi$ is

$$
\xi=C(T)^{-1} \eta(T)=e^{-0.5551 z-0.2040}
$$

where $z$ is a standard normal random variable. Hence, $E(\xi)=0.9512$ and $V^{2}(\xi)=0.5714$. By Equation (11),

$$
\left\{\begin{array}{l}
\lambda=7.5427 \mu-5.8269 \\
\rho=-5.8269 \mu+6.1256
\end{array}\right.
$$


The mean-variance optimal portfolio is, by Equations (10),

$$
W_{m}=(3.7764 \mu-2.9135)+(-2.9135 \mu+3.0628) \xi
$$

Assuming the utility function is logarithmic, then the optimal terminal wealth is (without loss of generality let $W_{0}=1$ )

$$
W_{u}=W_{0} \xi^{-1}=\xi^{-1}
$$

See Cox and Huang (1989) for a derivation of this. The intersection points, $\xi_{1}$ and $\xi_{2}$, are given by the quadratic equation

$$
(-2.9135 \mu+3.0628) \xi_{*}^{2}+(3.7764 \mu-2.9135) \xi_{*}-1=0,
$$

and whose solutions are

$$
\left\{\begin{array}{l}
\xi_{1}(\mu)=\frac{-5.8269+7.4527 \mu-\sqrt{82.9576-134.5163 \mu+56.8923 \mu^{2}}}{2(5.8269 \mu-6.1256)} \\
\xi_{2}(\mu)=\frac{-5.8269+7.4527 \mu+\sqrt{82.9576-134.5163 \mu+56.8923 \mu^{2}}}{2(5.8269 \mu-6.1256)} .
\end{array}\right.
$$

For given $\mu$, the probability that the mean-variance model outperforms the growth optimal strategy is

$$
\int_{\xi_{1}}^{\xi_{2}} \frac{1}{\sqrt{2 \pi} \kappa} \exp \left\{-\frac{\left(\ln x+r+\frac{1}{2} \kappa^{2}\right)^{2}}{2 \kappa^{2}}\right\} \cdot \frac{1}{x} d x
$$

Using the first order condition indicates that the numerical solution of the optimal $\mu$ is

$$
\mu \approx 2.10
$$

which means that, for this specific investment environment, investors should set the target wealth to be about double the initial wealth to maximize the probability of surpassing the growth optimal strategy. The maximum probability exceeds $80 \%$, i.e., eight out of ten times 
the mean-variance model will beat the growth optimal strategy under the assumption of lognormal asset prices. Figure 4 depicts the probabilities corresponding to different choices of $\mu$.

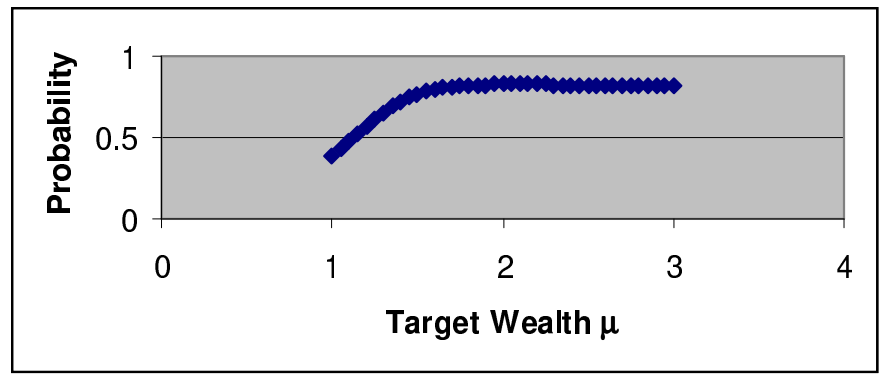

Figure 4: The probability superior to the growth optimal strategy

Remark. Since the logarithmic utility has an expected portfolio return $e^{\left(r+\kappa^{2}\right) T}$ which is dominant in the long run (as $T \longrightarrow \infty$ ), the logarithmic utility will have a higher chance of beating the mean-variance analysis for the long investment horizon. On the other hand, a mean-variance analysis is more sensitive to the volatility of asset returns than the logarithmic utility approach by examining the expressions of the optimal portfolio values. This leads to the assertion that the logarithmic utility may have a high probability of beating a mean-variance model when the market investment environments are changed to a long investment horizon and/or a moderately low price for risk (a small $\kappa$ ). See discussion on this in Hakansson and Ziemba (1995). 


\section{The Optimal Value Process and The Optimal Policy}

The partial differential equation approach has been extensively used for the valuation of contingent claims based on the assumption of a Markovian structure for the underlying assets. Usually, a parabolic equation with boundary conditions has to be solved. We adopt this approach to calculate the optimal portfolio value for each time $t$ which will derive the optimal portfolio policies by comparing the corresponding coefficients of the stochastic differential equations in wealth.

\section{A. The Optimal Value Process}

By the martingale representation theorem, any discounted martingale can be replicated by the market's primitive assets. Since a martingale is almost surely determined by the ending term of the martingale process, the optimal wealth $W(t)$ at time $t$ must be

$$
\begin{aligned}
W(t) & =C(t) E^{Q}\left(C(T)^{-1} W \mid \mathcal{F}_{t}\right) \\
& =C(t) \eta(t)^{-1} E\left(C(T)^{-1} \eta(T) W \mid \mathcal{F}_{t}\right) \\
& =\xi(t)^{-1} E\left(\xi(T) W \mid \mathcal{F}_{t}\right)
\end{aligned}
$$

The vector process $(C(t), S(t), \xi(t))$ is a Markovian process by the definition of $\eta(t)$ and the asset price model setting in (1), but $\xi(t)$ is not a discounted martingale, therefore, it is not replicable. However $\zeta(t)=\xi(t)^{-1}$ is a discounted martingale, usually called an inflator process, since

$$
d \zeta(t)=\zeta(t)\left[r(t) d t+\kappa(t)^{\top} d z^{Q}(t)\right]
$$


Hence, $(C(t), S(t), \zeta(t))$ are jointly a markovian process. The following corollary can be derived.

Corollary 2: With the setting of the underlying asset prices in (1), the optimal wealth at time $t$ is a function of $(C(t), S(t), \zeta(t))$.

\section{B. The Optimal Portfolio Policy}

Since $W(t)$ is a function of $(C(t), S(t), \zeta(t))$ at time $t$ by Corrolary 2, one needs only look for a function $F(t, C, S, \zeta)$ such that $W(t)=F(t, C(t), S(t), \zeta(t))$ satisfies the boundary condition. Assuming $F$ is continuously differentiable in $t$ and $C$ and twice continuously differentiable in $S$ and $\zeta$, Ito's formula and the comparison of the differential equation with (6) yield

$$
\left\{\begin{array}{l}
F_{t}+r(t) C(t) F_{C}+F_{S}^{\top} b(t)+r(t) \zeta(t) F_{\zeta}+\frac{1}{2} \operatorname{tr}\left(F_{S S} \sigma(t) \sigma(t)^{\top}\right) \\
\quad+\frac{1}{2} \zeta(t)^{2} \kappa(t)^{\top} \kappa(t) F_{\zeta \zeta}+F_{S \zeta}^{\top} \sigma(t) \kappa(t)=r(t) F \\
F_{S}^{\top} \sigma(t)+F_{\zeta} \zeta(t) \kappa(t)^{\top}=\theta(t)^{\top} \sigma(t)
\end{array}\right.
$$

where $\operatorname{tr}()$ is the trace function of a square matrix and $F$. and $F$.. stand for partial derivatives. Since the terminal value of the optimal portfolio $W=\frac{1}{2} \lambda+\frac{1}{2} \rho \zeta(T)^{-1}$ is implicitly a function of $r(T), b(T), \sigma(T)$ and $\zeta(T)$, the function $F(t, C, S, \zeta)$ is given by the partial differential equation stated in Theorem 3 
Theorem 3: The optimal wealth $F(t, C, S, \zeta)$ is given by the solution to the following partial differential equation and the boundary condition.

$$
\left\{\begin{array}{l}
F_{t}+r C F_{C}+F_{S}^{\top} b+r \zeta F_{\zeta}+\frac{1}{2} \operatorname{tr}\left(F_{S S} \sigma \sigma^{\top}\right)+\frac{1}{2} \zeta^{2} \kappa^{\top} \kappa F_{\zeta \zeta}+F_{S \zeta}^{\top} \sigma \kappa=r F \\
F(T, C, S, \zeta)=\frac{1}{2} \lambda+\frac{1}{2} \rho \zeta^{-1}
\end{array}\right.
$$

Therefore, the optimal portfolio policy is

$$
\left\{\begin{array}{l}
\theta(t)=F_{S}+F_{\zeta} \zeta(t)\left(\sigma(t)^{\top}\right)^{-1} \kappa(t) \\
\alpha(t)=C(t)^{-1}\left(W_{t}-\theta(t)^{\top} \cdot S(t)\right)
\end{array}\right.
$$

To understand the optimal portfolio policy better, we provide an intuitive interpretation. Since $\zeta(t)$ is a discounted Q-martingale, let $\theta^{\zeta}(t)$ be the hedging portfolio of $\zeta(t)$ in the risky assets, i.e.

$$
d \zeta(t)=r(t) \zeta(t) d t+\theta^{\zeta}(t)^{\top} \sigma(t) d z^{Q}(t)
$$

Comparing (23) with (20) yields

$$
\theta^{\zeta}(t)=\zeta(t)\left(\sigma(t)^{\top}\right)^{-1} \kappa(t)
$$

The optimal policy in (22) becomes

$$
\left\{\begin{array}{l}
\theta(t)=F_{S}+F_{\zeta} \cdot \theta^{\zeta}(t) \\
\alpha(t)=C(t)^{-1}\left(W(t)-\theta(t)^{\top} \cdot S(t)\right)
\end{array}\right.
$$

which can be interpreted as follows. If $\zeta(t)$ is considered to be a dynamic mutual fund, the optimal portfolio policy can be constructed by investing $F_{S}$ units in the assets $S$ and $F_{\zeta}$ units in the dynamic mutual fund $\zeta$. To implement this strategy, one needs only to 
synthesize the dynamic mutual fund $\zeta(t)$ and combine the two hedging portfolios (i.e., the $\Delta$ strategies) to obtain the optimal investment portfolio. This interpretation will become more intuitive and clearer in light of the special case discussed in the next section.

\section{A Special Case}

In this section we consider a special case of the model in which the asset prices jointly follow a multiperiod Brownian motion and the riskless rate is a constant throughout the investment horizon. This assumption implies that

$$
r(t)=r, \quad \sigma(t)=\left(\begin{array}{cccc}
S_{1}(t) & & & \\
& & & \\
& S_{2}(t) & & \\
& & \ddots & \\
& & & \\
& & S_{n}(t)
\end{array}\right) \sigma, \quad b(t)=\left(\begin{array}{c}
b_{1} S_{1}(t) \\
b_{2} S_{2}(t) \\
\vdots \\
\\
\\
\end{array}\right.
$$

where $r, \sigma, b$ are constant matrices. With these conditions, $\kappa(t)$ becomes a constant vector and

$$
\begin{aligned}
E\left(C(T)^{-1} \eta(T)\right) & =E\left(e^{-\kappa^{\top} z(T)-\left(r+\frac{1}{2} \kappa^{\top} \kappa\right) T}\right) \\
& =e^{-r T} \\
V^{2}\left(C(T)^{-1} \eta(T)\right) & =E\left(e^{-2 \kappa^{\top} z(T)-\left(2 r+\kappa^{\top} \kappa\right) T}\right)-e^{-2 r T} \\
& =e^{-2 r T}\left(e^{\kappa^{\top} \kappa T}-1\right) .
\end{aligned}
$$

Therefore,

$$
\begin{aligned}
\lambda & =\frac{2 \mu e^{\kappa^{\top} \kappa T}-2 W_{0} e^{r T}}{e^{\kappa^{\top} \kappa T}-1} \\
\rho & =\frac{2 W_{0} e^{2 r T}-2 \mu e^{r T}}{e^{\kappa^{\top} \kappa T}-1} .
\end{aligned}
$$




\section{A. The Closed Form Solution}

Since $\lambda$ and $\rho$ are constants throughout the investment horizon, $W=W(T)$ is only a function of $\zeta(T)$. Then we try to look for a function $F(t, \zeta)$ of $t$ and $\zeta$ such that it derives the optimal wealth process and the optimal portfolio policies. The partial differential equation (21) becomes

$$
\left\{\begin{array}{l}
F_{t}+r \zeta F_{\zeta}+\frac{1}{2} \zeta^{2} \kappa^{\top} \kappa F_{\zeta \zeta}=r F \\
F(T, \zeta)=\frac{1}{2} \lambda+\frac{1}{2} \rho \zeta^{-1}
\end{array}\right.
$$

Solving this equation yields

Theorem 4: The partial differential equation (28) has a closed form solution

$$
F(t, \zeta)=\frac{1}{2}\left(\lambda e^{-r(T-t)}+\rho \zeta^{-1} e^{\left(\kappa^{\top} \kappa-2 r\right)(T-t)}\right)
$$

The optimal portfolio policy at time $t$ is

$$
\left\{\begin{array}{l}
\theta(t)=\left(\frac{1}{2} \lambda e^{-r(T-t)}-W(t)\right) I_{S}^{-1}\left(\sigma^{\top}\right)^{-1} \kappa \\
\alpha(t)=C(t)^{-1}\left(W(t)-\theta(t)^{\top} S(t)\right),
\end{array}\right.
$$

where $I_{S}$ is the diagonal matrix with asset prices, $S(t)$, as the entries.

Proof of Theorem 4. The first and second order derivatives of $F(t, \zeta)$,

$$
\begin{aligned}
F_{t} & =\frac{1}{2} \lambda r e^{-r(T-t)}-\frac{1}{2} \rho \zeta^{-1}\left(\kappa^{\top} \kappa-2 r\right) e^{\left(\kappa^{\top} \kappa-2 r\right)(T-t)} \\
F_{\xi} & =-\frac{1}{2} \zeta^{-2} \rho e^{\left(\kappa^{\top} \kappa-2 r\right)(T-t)} \\
F_{\xi \xi} & =\rho \zeta^{-3} e^{\left(\kappa^{\top} \kappa-2 r\right)(T-t)},
\end{aligned}
$$


satisfy Equation (28) and the boundary condition. So, $F(t, \zeta)$ is the solution to the partial differntial equation. By Ito's formula,

$$
d F(t, \zeta(t))=r F(t, \zeta(t)) d t-\frac{1}{2} \rho \zeta^{-1} e^{\left(\kappa^{\top} \kappa-2 r\right)(T-t)} \kappa^{\top} d z^{Q}(t)
$$

which derives the optimal strategy (25) by comparing with the wealth dynamics (6) and using (29).

\section{Concluding Remarks}

Applying martingale analysis, this paper derives the mean-variance efficient frontier and optimal portfolio policies for dynamic investments. The efficient frontier is uniquely determined by the mean and the standard deviation of the contingent state price. The efficient frontier is also a ray which intersects the vertical axis corresponding to the mean return at the return of the risk free asset with slope equal to the ratio of the mean and the standard deviation of the contingent price.

To solve a dynamic investment model using the mean-variance criterion, one needs only to obtain the probability distribution of the contingent state price since, for a given distribution of the contingent state price, the optimal portfolio value is a linear function (with negative slope) of the outcome of the contingent state price. After identifying the optimal portfolio value, one can apply a replication procedure (either a partial differential approach as in this paper or a stochastic programming method in Zhao and Ziemba (2000)) to derive

the optimal portfolio strategies. With lognormal asset prices, using a dynamic mutual fund as an auxiliary asset a closed form solution of the optimal portfolio strategies exists. 
To show the important role of mean-variance analysis in making sound investment decisions, this paper compares, state by state, the optimal values obtained from both meanvariance and expected utility models. The mean-variance analysis is superior to the expected utility if the outcome of the contingent state price is near its mean and inferior to the expected utility model if the outcome is in the tails. An interesting question is: what is the optimal target wealth such that the mean-variance criterion will be superior to a given expected utility model with maximum probability? To perform the analysis, we used a data from the Dow Jones Industrial Average from March 7, 1977, to February 7, 2000, to calibrate the asset price model. For a one-year investment horizon, the mean-variance return will beat the growth optimal (logarithmic utility) return by more than an $80 \%$ chance if the wealth target is set to be about double the initial wealth. 


\section{References}

Cox, J., and C. Huang, 1989, Optimal Consumption and Portfolio Polices When Asset Prices Follow a Diffusion Process, Journal of Economic Theory, 49, 33-83.

Grauer, R. R., 1981, A Comparison of Growth Optimal and Mean Variance Investment Policies, Journal of Financial and Quantitative Analysis, 16, 1-21.

Grauer, R. R., and N. H. Hakansson, 1993, On the Use of Mean-Variance and Quadratic Approximations in Implementing Dynamic Investment Strategies: A Comparison of the Returns and Investment Policies, Management Science, 39, 856-871.

Hakansson, N. H., 1971, Capital Growth and the Mean-Variance Approach to Portfolio Selection, Journal of Financial and Quantitative Analysis, 6, 517-557.

Hakansson, N. H., and W. T. Ziemba, 1995, Capital Growth Theory, in: Finance, (eds.), R. Jarrow, V. Maksimovic and W.T. Ziemba, North-Holland, pp. 61-86.

Harrison, M., and D. Kreps, 1979, Martingale and Multiperiod Securities Markets, Journal of Economic Theory, 20, 382-408.

Harrison, M., and S. Pliska, 1981, Martingales and Stochastic Integrals in the Theory of Continuous Trading, Stochastic Process Appl., 11, 215-260.

Kroll, Y., H. Levy, and H. Markowitz, 1984, Mean-Varaince versus Direct Utility Maximization, Journal of Finance, 39, 47-75.

Levy, H., and H. Markowitz, 1979, Approximating Expected Utility by a Function of Mean and Variance, American Economic Review, 69, 308-317.

Markowitz, H., 1952, Portfolio Selection, Journal of Finance, 7(1), 77-91.

Merton, R., 1969, Lifetime Portfolio Selection Under Uncertainty: the Continuous Time Case, Review of Economic Statistics, 51, 247-257.

- 1971, Optimal Consumption and Portfolio Rules in a Continuous -Time Model, Journal of Economic Theory, 3, 373-413. 
Pliska, S., 1986, A Stochastic Calculus Model of Continuous Trading: Optimal Portfolio, Mathematics of Operations Research, 11, 371-382.

Samuelson, P., 1967, General Proof that Diversification Pays, Journal of Financial and Quantitative Analysis, 3(3), 1-13.

Tobin, J., 1958, Liquidity Preference as Behaviour Towards Risk, Review of Economic Statistics, 25(2), 65-86.

Zhao, Y., U. Haussmann, and W. Ziemba, 2000, A Dynamic Investment Model with a Minimum Attainable Wealth Requirement, submitted to The Review of Financial Studies.

Zhao, Y., and W. T. Ziemba, 2000, Determining Risk Neutral Probability and Optimal Policies in a Dynamic Investment model in the Presence of Trading Frictions, submitted to the Journal of Financial and Quantitative Analysis.

Ziemba, W., and J. Mulvey, (eds.), 1998, World Wide Asset and Liability Modeling. Princeton University Press.

Ziemba, W., C. Parkan, and R. Brook-Hill, 1974, Calculation of Investment Portfolio with Risk Free Borrowing and Lending, Management Science, 21(2), 209-222.

Ziemba, W., and R. Vickson, (eds.), 1975, Stochastic Optimization Models in Finance. Academic Press. 